\title{
Publisher Correction: Genomes of 13 domesticated and wild rice relatives highlight genetic conservation, turnover and innovation across the genus Oryza
}

Joshua C. Stein, Yeisoo Yu, Dario Copetti, Derrick J. Zwickl, Li Zhang (D), Chengjun Zhang (D), Kapeel Chougule, Dongying Gao, Aiko Iwata, Jose Luis Goicoechea, Sharon Wei, Jun Wang, Yi Liao, Muhua Wang, Julie Jacquemin, Claude Becker (D), Dave Kudrna, Jianwei Zhang, Carlos E. M. Londono, Xiang Song, Seunghee Lee, Paul Sanchez, Andrea Zuccolo D, Jetty S. S. Ammiraju, Jayson Talag, Ann Danowitz, Luis F. Rivera, Andrea R. Gschwend, Christos Noutsos, Cheng-chieh Wu, Shu-min Kao, Jhih-wun Zeng, Fu-jin Wei, Qiang Zhao, Qi Feng (D), Moaine El Baidouri, Marie-Christine Carpentier, Eric Lasserre D, Richard Cooke, Daniel da Rosa Farias, Luciano Carlos da Maia, Railson S. dos Santos, Kevin G. Nyberg, Kenneth L. McNally, Ramil Mauleon, Nickolai Alexandrov, Jeremy Schmutz, Dave Flowers, Chuanzhu Fan, Detlef Weigel, Kshirod K. Jena, Thomas Wicker, Mingsheng Chen, Bin Han, Robert Henry (D), Yue-ie C. Hsing, Nori Kurata, Antonio Costa de Oliveira, Olivier Panaud (D), Scott A. Jackson (D), Carlos A. Machado, Michael J. Sanderson, Manyuan Long (iD, Doreen Ware (iD and Rod A. Wing (iD)

Correction to: Nature Genetics https://doi.org/10.1038/s41588-018-0040-0, published online 22 January 2018.

This article was not made open access when initially published online, which was corrected before print publication. In addition, ORCID links were missing for 12 authors and have been added to the HTML and PDF versions of the article. 